\title{
High Fidelity Simulations of Flapping Wings Designed for Energetically Optimal Flight
}

\author{
Per-Olof Persson* \\ University of California, Berkeley, Berkeley, CA 94720-3840, U.S.A. \\ David J. Willis ${ }^{\dagger}$ \\ University of Massachusetts, Lowell, Lowell, MA 01854, U.S.A.
}

\begin{abstract}
A diversity of efficient solutions for flapping flight have evolved in nature; however, it is often difficult to isolate the key characteristics of efficient flapping flight from biological constraints. Rather than base micro aerial vehicle (MAV) design on natural flyers alone, we propose a multi-fidelity computational approach for analysis and design. At the lowest fidelity level, we use a wake-only energetics model that allows us to rapidly scan the global flapping kinematics for efficient kinematics and configurations. Following the wake-only design space characterization, we determine a series of candidate flapping wing geometries that can produce the desired wake characteristics. To do this, we have developed a quasiinverse wing design strategy that attempts to match the designed vehicle's wake-circulation distribution with that predicted by the energetics model. Using our modified-doublet lattice method, we are able to determine how to modulate wing twist and camber to produce the desired wake vorticity. Because the method assumes inviscid flow, we are able to derive a large number of candidate designs to produce the target wake; however, as we show in this paper, only some of the designs perform adequately in physically relevant viscous fluids. As such, we use a high order, Discontinuous Galerkin, Navier-Stokes solver to simulate and assess the candidate designs, and examine which geometries minimize flow separation, improve performance and increase efficiency. The focus of this paper is on the design and analysis of efficient flapping wings. We present an application of our framework to a MAV design that has similar characteristics as medium sized fruit bat. We examine candidate wing designs to illustrate how adjusting wing section camber may be more favorable than adjusting wing twist alone. We find that the angle the leading edge of the wing presents to the flow is critical to minimizing flow separation.
\end{abstract}

\section{Introduction}

Flapping wings present a challenging, yet potentially rewarding approach for achieving efficient flight at low Reynolds numbers and small size scales. Most natural flyers demonstrate impressive abilities to maneuver and migrate in a low-Reynolds number regime. These impressive flight qualities allow animals to forage, escape prey and to travel great distances. It is these qualities that are attractive to micro aerial vehicle (MAV) designers. Ideally, we would like to isolate the wing kinematics that permit these impressive flight qualities and integrate them into simpler wing motions and deformations; however, isolating and simplifying these kinematics from observations of nature alone is challenging. Since the majority of the flight time for most MAVs will be spent in cruise, we focus here on computational approaches for designing efficient flapping wings. While this may seem limiting, the challenges of highly maneuverable flight will likely derive naturally from the base flight platform.

One of the primary challenges of developing practical and efficient flapping wing micro aerial vehicles (MAVs) is the infinite aerodynamics performance design space. Flapping amplitude, frequency, local wing

\footnotetext{
${ }^{*}$ Assistant Professor, Department of Mathematics, University of California, Berkeley, Berkeley CA 94720-3840. E-mail: persson@berkeley.edu. AIAA Member.

${ }^{\dagger}$ Assistant Professor, Department of Mechanical Engineering, University of Massachusetts, Lowell, Lowell, MA. E-mail: David_Willis@uml.edu. AIAA Member.
} 
twist, local camber, and forward aft flapping all play a role in achieving efficient flight performance. While nature demonstrates several solutions for flapping kinematics, it is unclear which of these kinematics are aerodynamically functional and which are as a result of biological constraints. As such, while natural flyers provide a large degree of insight, mimicking these flyers may result in overly complex MAV designs.

Using high-fidelity tools to investigate all of the possible flapping parameters, while also satisfying flight equilibrium conditions (mean lift equals mean weight and mean drag equals mean thrust) is practically infeasible. One approach is to examine a multi-resolution approach while another would be to strategically mimic traditional wing design processes and implement low-fidelity computational models to assist with narrowing the flapping wing design space and confirm the design with higher fidelity tools. We choose the later strategy, and use a multi-fidelity framework that comprises a wake-only method, a quasi-doublet lattice method for inverse wing design and a high fidelity Navier-Stokes simulation. We have shown that our particular multi-fidelity approach is promising for both two-dimensional and three-dimensional settings such as thrust producing flapping foils ${ }^{1,2}$. We present a similar framework here for designing efficient wings and validating those designs at the highest fidelity level. In this paper we examine the design and characteristics of efficient three dimensional wings.

In this paper we examine the inverse design of flapping wings starting from an optimal circulation wake predicted using the low-fidelity energetics model..$^{3,4}$ We select a single flight speed and a single optimal wake and demonstrate the wing design process using the quasi-doublet lattice method. The inverse design is based on wing twist modulation of a prescribed wing geometry and sectional camber. The resulting wing designs and accompanying kinematics are examined using a high order Discontinuous Galerkin, Navier-Stokes method. The primary focus in this paper is the importance of viscous considerations in the inverse design process. In particular, we demonstrate this using adaptive wing leading edge geometry in a viscous fluid.

\section{Wake Only Energetics}

At the lowest fidelity level, we focus on the core global parameters of the wing design, (the flapping amplitude, frequency, power consumption, etc.) rather than the details of the geometry (wing twist, local camber, leading edge angle, etc.). This allows us to simplify the computational analysis and rapidly evaluate the flight performance of a large number of candidate designs and configurations. We use a wake-only energetics model ${ }^{3}$ that is based on a wake-only method, ${ }^{5}$ which is derived from a control volume approach. This wake-only method is used to determine the minimum-energy circulation distribution that achieves the prescribed vertical and horizontal forces. Using the method of Hall et al., ${ }^{5}$ we have developed a flight-forcebalance criteria and developed an energetics estimation tool. ${ }^{3}$ This wake-only energetics model predicts the power consumption as well as the optimal wing-beat frequency and amplitude for a range of flight speeds both for level flight as well as ascending and descending flight. An example energetics analysis for the MAV considered in this paper is shown in figures 1 and 2. The energetics and corresponding kinematics relationships for a medium sized MAV with a weight of 35 grams with a wing span of 38 centimeters are illustrated. In addition to the energetics and kinematics parameters, the wake-only method produces one critical piece of additional information - the wake circulation distribution. The wake circulation distribution indicates how the fluid in the wake of the animal changes momentum in order produce lift and thrust for the flapping wing. This wake circulation distribution is used as the target or goal wake in the inverse design process (Figure 3).

The energetics model is a powerful tool for predicting the general kinematics behavior of efficient flapping wings; however, a drawback for the MAV designer of this simple low-fidelity tool is the lack of wing geometric information. Our wake-only method assumes a lifting-line or simply a stick-figure wing. Little assumption is made about the wing planform, the wing twist or the wing camber, all of which become key geometric variables in the wing design process. The main goal of the remainder of this paper is to describe the process by which we develop a geometric description of the wing that is capable of generating the flight forces.

\section{Inverse Wing Design and Medium Fidelity Simulations}

The wake-only method provides a target wake shape and a target wake circulation distribution that corresponds to an energetically efficient wing. It is this wake circulation distribution that we use to develop a flapping wing geometry. We employ a modified doublet-lattice method ${ }^{6,7}$ to compute the wing shape that produces the desired circulation distribution during the wingbeat cycle. We parametrize the wing shape 


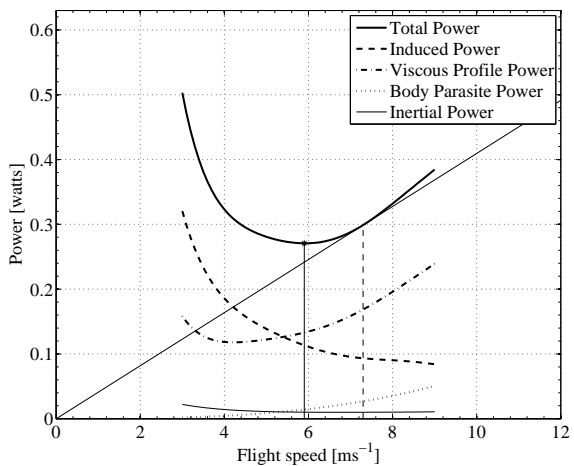

Figure 1. The bat total power, viscous power, induced power, and inertial power components as predicted using the wake-only energetics model. ${ }^{3,6,7}$
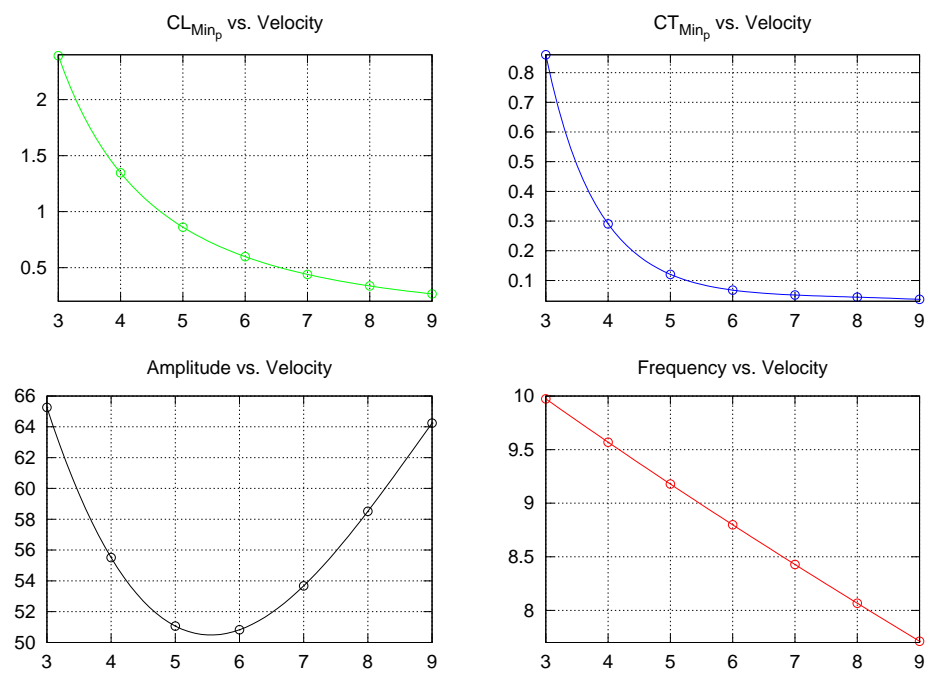

Figure 2. The lift, thrust, amplitude and frequency variations with velocity computed using the wake-only energetics model. ${ }^{3,6,7}$

using spanwise camber and spanwise twist information. Because of the limited number of elements in the discrete wake definition, the inverse design process at each time-step would be an under-constrained problem if both the camber and angle of attack are allowed to vary at each spanwise station of the geometry. Rather than allowing both camber and twist distribution to be modified, we prescribe the spanwise camber using a simple leading edge angle criterion and use our modified doublet-lattice method to modulate the wing twist so that the actual geometry sheds a comparable amount of vorticity into the wake as prescribed by the wake only optimum result. In the sections that follow we describe the process for defining the flapping geometry as well as the process in which the wing twist is modulated at each discrete step of the flapping cycle so that the desired energetics and flight characteristics are achieved.

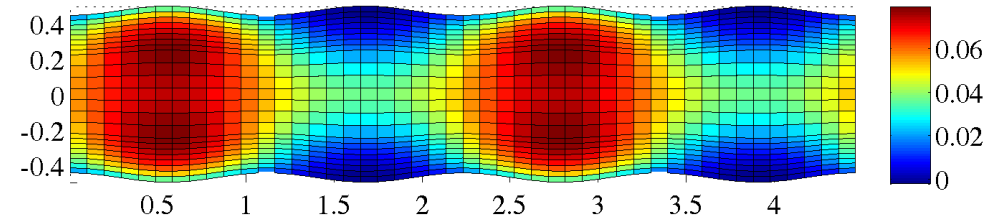

Figure 3. The target wake circulation distribution for the case-study presented in this paper. The wake geometry for a full flapping cycle is shown for two successive flapping periods, starting with the downstroke. The wake is color-coded based on the strength of the circulation shed at that spatial location. The wake only energetics method uses this as the geometry for evaluating optimal energetics performance. ${ }^{3,6,7}$ 


\section{III.A. Inverse Design: Wing Geometry Definition}

The wing geometry is best described by following the sequential steps that are used in the modified doublet lattice method: ${ }^{6,7}$

1. Define a reference wing planform: In this step we define the shape of the leading edge, the shape of the trailing edge, and the line about which spanwise wing stations will be rotated to generate the wing twist distribution. In our current implementation the wing leading and trailing edges are defined using parametrized quadratic lines that are defined by a three points along the semi-span leading and trailing edge respectively. This reference wing planform is shown in Fig. 4-a.

2. Modify spanwise sections using the mean-camberline: In this step we define the chordwise distribution of vertices at each spanwise station that make up the wing surface network. For each spanwise wing station, we define a two dimensional camberline. The wing camberline is defined using a Hermite Cubic Spline with the location and slope of the leading and trailing edges of the airfoil defining the camber (Fig. 4-b). In the examples presented in this paper, we define the leading edge slope with respect to the line segment that joins the leading and trailing edges, and we define the trailing edge slope as one half of the negative leading edge angle. At present, we have the option to prescribe the leading edge angle for the duration of the wingbeat cycle or, for more advanced cases, we define the leading edge angle based on the relative velocity at the local spanwise section.

3. Modify the wing sections to include the spanwise wing twist distribution: The next step in the geometry definition process is to locally twist the wing sections per the prescribed twist distribution. We simply rotate the local wing sections through the local twist angle at each spanwise station (Fig. 4-d \& e). This sectional rotation is performed about the prescribed rotation point. The wing twist and wing camber both act on the reference geometry. This means that the wing shape modifications are orthogonal to the wing reference plane and are not affected by the current flapping angle of the wing. This should improve the feasibility of realistically implementing these wings in MAV designs.

4. Rotate the wing through the flapping angle: The final step in the geometry definition is to rotate the wing through the prescribed flapping angle (Fig.4-f). At this stage, the wing is discretized into a collection of surface elements for the inviscid flow solvers.

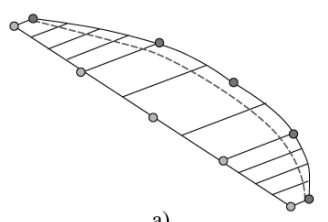

a)

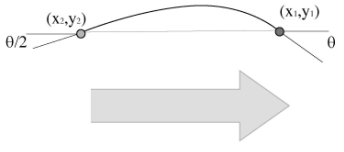

b)

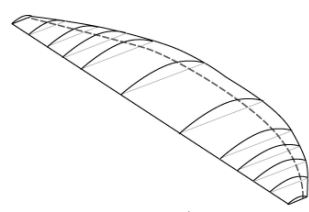

c)
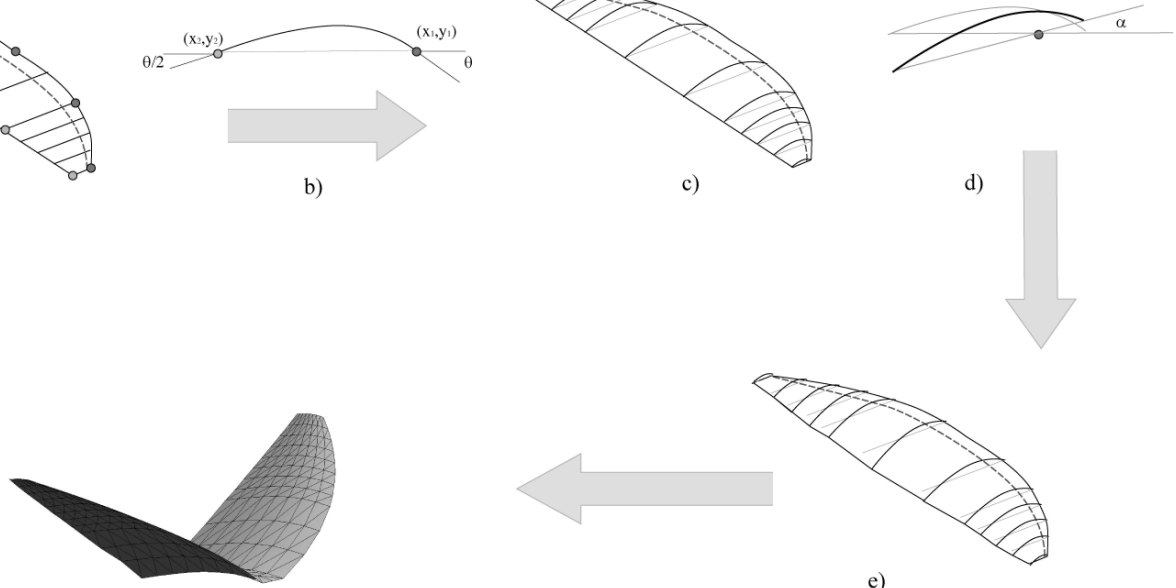

e)

Figure 4. The wing geometry definition process. (a) The leading edge, trailing edge and local twist rotation lines define the wing planform. (b) A two point Hermite Spline is used to define a cambered wing section (c) The cambered airfoil section is integrated into the appropriate spanwise section of the wing. (d \& e) Individual spanwise stations of the wing are rotated through the local incidence angle about the pre-defined rotation point. (f) The wing is rotated through the current flapping angle.

By using this process for generating our geometry, we are able to automatically, and efficiently define new wing shapes with a variety of wing leading edge angles and wing twist distributions. This generic geometry definition process is used in the modified-doublet lattice method to permit rapid geometry modifications and to evaluate candidate geometries at different timesteps. In our implementation of the geometry generator, 
we simply input the points to define the wing planform, the spanwise location of spanwise stations (defined by the optimal wake-only energetics solution), the spanwise section leading (and trailing) edge angles, the spanwise section twist angle, and the current flapping angle. The output of our geometry generation tool is a collection of vertices, a connectivity array and a trailing edge line along which vorticity will be shed. This minimum amount of information can describe a large variety of possible flapping wing geometries, and can be easily modified.

\section{III.B. Inverse Design : Modified Doublet Lattice Method}

In this paper we only briefly describe our quasi-inverse wing design approach ${ }^{6,7}$ and highlight the differences between the present method and previous versions. At the core of our wing design methodology is a modified doublet lattice method which solves the following boundary integral equation for potential flow:

$$
\begin{aligned}
\nabla\left(\phi\left(x, x^{\prime}\right)\right) \cdot \hat{n} & =\underbrace{\frac{\partial}{\partial n}\left(\iint_{S W} \mu_{\text {wing }} \frac{\partial}{\partial n^{\prime}}\left[\frac{1}{\left\|x-x^{\prime}\right\|}\right] d S_{\text {wing }}\right)}_{I_{\mathrm{wing}}} \\
& +\underbrace{\frac{\partial}{\partial n}\left(\iint_{S W_{\mathrm{bw}}} \mu_{W_{\mathrm{bw}}} \frac{\partial}{\partial n^{\prime}}\left[\frac{1}{\left\|x-x^{\prime}\right\|}\right] d S_{\mathrm{bw}}\right)}_{I_{\mathrm{buffer}-\text { wake }}} \\
& +\underbrace{\frac{\partial}{\partial n}\left(\iint_{S W_{\mathrm{kw}}} \mu_{W_{\mathrm{kw}}} \frac{\partial}{\partial n^{\prime}}\left[\frac{1}{\left\|x-x^{\prime}\right\|}\right] d S_{\mathrm{kw}}\right)}_{I_{\mathrm{known}-\text { wake }}} .
\end{aligned}
$$

The boundary integral equation represents the influence of the different doublet distributions in the model on the overall potential flow description - in this case, the influence of doublet sheets that represent the wing and the wake. The wing doublet distribution influence or the wing bound vorticity is represented in the first integral term $\left(I_{\text {wing }}\right)$ in the above equation. The wing doublet strengths are unknown at each timestep and are determined for each geometry that is tested in the process. The second integral over the buffer wake $\left(I_{\text {buffer-wake }}\right)$ represents the shed wake vorticity in the region immediately adjacent to the trailing edge for the current timestep, and is typically determined using a Kutta-condition during the solution process. We denote this as the buffer wake since it interfaces the current wing geometry with the shed wake from previous timesteps. While this portion of the wake is a small component of the overall shed vorticity, it is a critical component, since this is the portion of the wake that we are interested in matching to the target wake from the optimal energetics solution. In our design method, the buffer wake is unknown for each geometry that is examined, however, after the solution is determined, then the buffer wake is the key component of the simulation that is compared with the optimal wake circulation distribution. The final doublet influence in the above equation represents the known wake $\left(I_{\text {known-wake }}\right)$. In our case, this is the known optimal wake's influence on the domain for all of the discrete timesteps prior to the current timestep.

In a traditional doublet lattice method the wing doublet strength $\left(\mu_{\text {wing }}\right)$, and the unknown wake doublet strength for that timestep $\left(\mu_{W_{\mathrm{tw}}}\right)$ are determined at each timestep. This unknown doublet strength is determined using a Kutta condition that approximately satisfies the smooth trailing edge flow condition. In our modified doublet lattice method, we still solve the traditional doublet lattice method at each timestep; however, rather than advancing in time following our solution, we iterate and modulate the wing twist until the target-wake matches the desired optimal wake. Therefore, the key difference between our doublet lattice implementation and the traditional doublet lattice, is that we use the difference between the current buffer wake doublet strength and the target doublet strength to modify the wing twist distribution until the difference between the two is leas than a prescribed tolerance.

The primary result of our quasi-inverse doublet lattice method is a surface representation of the wing geometry. In addition, we can also output the time-dependent global wing twist angle at any spanwise station, the spanwise camber distribution, the spanwise variation of leading edge angle, and the wake strength and shape that was achieved by the designed wing. These metrics can all provide insight into the wing design, especially at later stages when viscous effects are included. 


\section{III.C. Medium Fidelity Simulations: FastAero}

We use our potential flow simulation tool, FastAero to simulate the potential flow around the morphing wing geometry. We use FastAero because it is a rapid evaluation, computational aerodynamics tool, that is well suited to modeling unsteady potential flow around flapping wings. Again, we only briefly describe the method and refer the reader to previous publications. ${ }^{8}$ As a potential flow method, the underlying flow physics assumptions are an inviscid, irrotational and incompressible flow. These flow physics assumptions are of key concern in our multi-fidelity strategy for analyzing and designing flapping vehicles. As such, much of our recent attention, including this paper, is aimed at examining the limits of validity of these simpler fluid models. ${ }^{2}$

FastAero is a boundary element method or panel method for modeling both two and three dimensional unsteady potential flow around morphing geometries. The method has the flexibility to solve the potential flow around bodies with finite thickness (the Dirichlet formulation) or infinitely thin surfaces (the Neumann formulation). FastAero was developed for efficiently modeling unsteady potential flow. FastAero has several key features to enable rapid unsteady flow modeling. First, iterative linear system solution methods ${ }^{9}$ are accelerated using approximate matrix-vector products including precorrected-FFT ${ }^{10}$ and the fast-multipoletree methods. ${ }^{11,12}$ Second, the source and doublet strengths are represented using linear basis functions across the surface elements, with the boundary integral equation being satisfied using a weighted residual method. Because we use linear basis functions, we are able to discretize the surface using unstructured meshes composed of triangles. In addition, the linear basis functions simplify the surface velocity and pressure computations. Third, we use a vortex particle method to represent the wake vorticity distribution. The vortex particle method enables a simple wake convection process in which wake roll-up and wake-body intersections are handled in a more automatic manner than traditional wake-sheet-body-panel intersection methods.

\section{High Fidelity Simulations of Flapping Wings}

\section{IV.A. Discontinuous Galerkin Arbitrary Lagrangian-Eulerian (ALE) Navier-Stokes: 3DG}

For high fidelity verification of our wing designs, we use the $3 D G$ solver which is a high-order Discontinuous Galerkin method (DG) method with tetrahedral mesh elements and nodal basis functions. High order methods are advantageous for applications requiring low numerical dispersion and high time accuracy. The DG method produces stable discretizations of the convective operator for any order discretization, thus avoiding the need for additional stabilization or filtering. Here, we use nodal basis and polynomial orders $p=3$. The viscous terms are discretized using the Compact Discontinuous Galerkin (CDG) method ${ }^{13}$ which leads to optimal accuracy, is compact, and generates sparser matrices than the alternative existing methods. For the time-integration, we use a 3-stage, 3rd order accurate Diagonally Implicit Runge-Kutta (DIRK) method. ${ }^{14}$

\section{IV.B. Geometry and Reference Mesh}

In order to obtain maximum geometrical flexibility, the compressible Navier-Stokes equations are discretized on unstructured meshes of tetrahedra. Due to the high level of resolution provided by the high order methods, we are able to resolve laminar flows up to $\operatorname{Re} \sim 2-4 \cdot 10^{4}$ using isotropic meshes. In this work, we use a lower Reynolds number of 3,000, to ensure that the flows are resolved and that our simulations are converged.

For the computational domain, we use the symmetry of the problem and consider one wing only. The outer boundary is a half-cylinder of radius 2 and length 4 . The surfaces are meshed in a parameter space using the DistMesh mesh generator, ${ }^{15}$ and the volume mesh is created using a Delaunay refinement based mesh generator. ${ }^{16}$ The resulting mesh has about 50,000 tetrahedra, which corresponds to 1 million high-order nodes of degree $p=3$ and 5 million degree of freedom for the Navier-Stokes equations.

\section{IV.C. Arbitrary Lagrangian-Eulerian (ALE) Formulation}

To account for the moving and the deforming domains, we use the mapping based Arbitrary LagrangianEulerian (ALE) formulation proposed by Persson et al. ${ }^{17}$ It can be accurate to arbitrary orders in both space and time. The so-called Geometric Conservation Law is satisfied using an additional equation that is used to compensate for the numerical integration errors. 

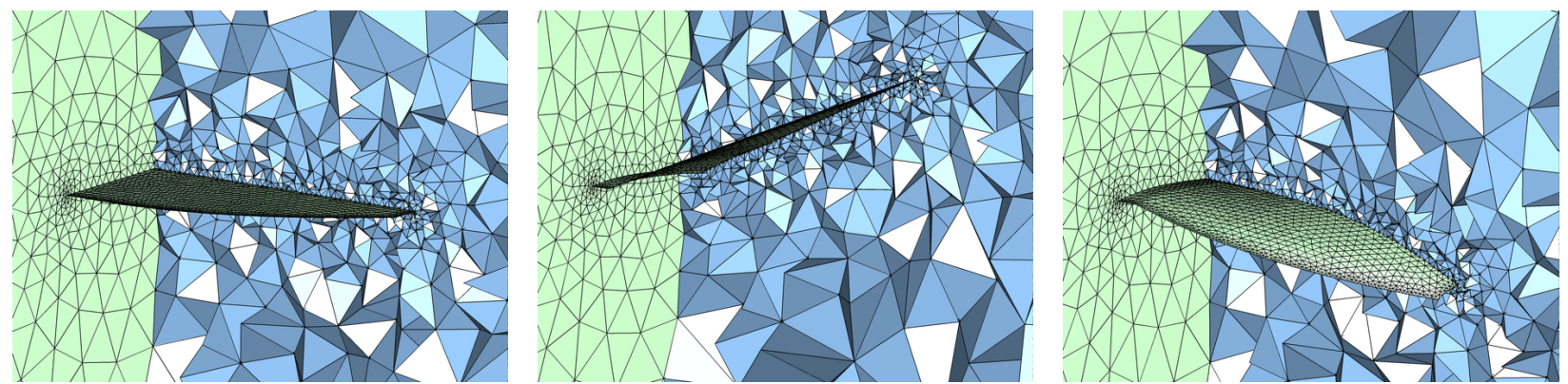

Figure 5. Mesh deformation using nonlinear elasticity. The reference mesh (left) is deformed as the wing surface is moved (middle and right).

The formulation requires that the deformations are prescribed either explicitly or indirectly as a mapping $x=x(X, t)$ between the reference and the physical space. Here, since the flapping wings are computed numerically, we also compute the mappings numerically. First, we obtain well-shaped deformed meshes for each instance of time in the DIRK scheme, using an elasticity analogy. Then we compute numerically the deformation gradient $\partial x / \partial X$ and the grid velocity $\partial x / \partial t$.

\section{IV.D. Mesh Deformation using Nonlinear Elasticity}

As the wing deforms according to the specified deformation, the entire tetrahedral mesh mustbe deformed in a smooth way such that the corresponding mapping $x(X, t)$ is well-behaved and, in particular, does not invert. We use a nonlinear elasticity analogy for this, similar to the one we proposed in Ref. 18 for generation of curved meshes. The reference mesh is considered an undeformed solid, and as the wing deforms we compute equilibrium configurations for the entire domain. Our nonlinear neo-Hookean formulation is discretized using a standard finite element method with elements of the same degree as the fluid solver (that is, $p=3$ ). It is highly resistant to element inversion, and an adaptive Newton method with homotopy in the boundary deformation is used to solve the discretized equations.

An example of the computed mesh deformation is shown in Fig. 5, with the reference mesh and two deformed meshes in their equilibrium configurations. These calculations are done as a preprocessing step, and the computational cost is negligible compared to the cost of the fluid solver.

\section{Case-Study: Flapping wing design for a bat-like flapping wing MAV}

We present results for a single operating point of a bat-like MAV design. First we invoke the energetics model to determine the kinematics of energetically efficient flight across a range of flight velocities. The general size characteristics of a medium sized fruit bat, Cynopertus brachyotis, were adopted as the basis of our wing design problem. To simplify the analysis for this case study, we focus exclusively on a flight velocity of $7 \mathrm{~m} / \mathrm{s}$ which corresponds to an operational point that lies between the predicted minimum power velocity and the maximum range velocity for this geometry. ${ }^{3,6,7}$ The energetics results for this bat-like geometry have been examined in previous publications, with good agreement with experimental observations. ${ }^{3,6,7}$

We examine 4 different flapping wing designs outlined in table 1. Each of these candidate wings are designed in the modified doublet lattice method to achieve exactly the same aerodynamics performance, namely, matching the target wake. While the performance of these wings in inviscid flow is expected to be quite similar, viscous effects, such as separation are expected to generate differences in actual performance between the different wings.

\begin{tabular}{|c|c|c|}
\hline \hline Case Id & Leading Edge Angle & Characteristics \\
\hline \hline Case 1 & $0^{\circ}$ & Wing sections have no camber. \\
Case 2 & $10^{\circ}$ & Fixed camber, $10^{\circ}$ leading edge angle, $5^{\circ}$ trailing edge angle. \\
Case 3 & $20^{\circ}$ & Fixed camber, $20^{\circ}$ leading edge angle, $10^{\circ}$ trailing edge angle. \\
Case 4 & Dynamic & Leading edge angle aligned with flow throughout the wingbeat cycle. \\
\hline \hline
\end{tabular}

Table 1. The variety of leading edge geometries considered in our study 


\section{Results and Discussion}

\section{VI.A. General Comparisons: Surface Pressure Distributions and Viscous Flow Simulations}

Figure 7 illustrates the FastAero predicted pressure differential distribution on the wing planform for the different leading edge conditions. For case 1, we notice that the lack of wing camber results in strong suction pressure peaks at the leading edge of the wing for the duration of the downstroke. This strong pressure differential at the leading edge of the wing results in the flow experiencing a strong adverse pressure gradient across the remainder of the chordwise section, suggesting flow separation is likely across the entire leading edge for the duration of the downstroke. During the upstroke, the pressure differential is much more benign due to the relatively inactive upstroke, and as such, we expect little or no separation. These suspicions are confirmed in the Navier-Stokes simulations for case 1 (figure 6), where a large leading edge vortex (LEV) forms over the suction side of the wing during the first third of the downstroke, followed by a subsequent shedding of the LEV and sustained flow separation through the remainder of the downstroke. The upstroke is characterized by attached flow and relatively little active momentum transfer to the fluid. The flow separation during the downstroke will adversely impact the desired efficiency characteristics, and as a result, this wing design is less attractive.

As a result of the adverse characteristics associated with the uncambered wing in case 1, we designed several wings with camber. Camber was introduced as a means to examine the impact of the relative angle between the leading edge and the flow on separation. Initially, we examined constant camber wings with fixed leading edge angles of $10^{\circ}$ and $20^{\circ}$, as case 2 and case 3 respectively. The FastAero pressure differential distributions illustrate the effect of camber on flapping wing aerodynamics. We observe a noticeable reduction in the leading edge suction peak. This reduction in the pressure peak at the leading edge is accompanied by a rear-ward redistribution of the pressure differential. In case 3, where camber is extreme, we see the maximum suction pressure occurs near the mid-chord section of the wing. The pressure recovery in both cases is much more gradual, with a correspondingly reduced adverse pressure gradient. This inviscid prediction of the pressure differential distribution suggests that the actual physical flow will be better behaved around these geometries. This suspicion is also confirmed in the Navier-Stokes results shown in figure 6. The viscous flow simulations predict little suction side separation for both cases 2 and 3. We hypothesize that this is primarily due to the smoother leading edge angle that is presented to the flow. The camber effectively aligns the leading edge of the wing with the onset flow. In case 3, where the leading edge angle is twenty degrees, we suspect that the stagnation streamline attaches to the wing on the suction side of the wing. This results in the flow on the suction surface of the wing being well-behaved; however, the flow must pass over the leading edge of the wing to reach the pressure side of the wing. This results in a non-suction pressure peak at the leading edge which will promote flow separation on the underside of the wing. We can see from the Navier-Stokes results (figure 6) that there are unsteady structures characteristic of separation on the wing underside and in the wake in case 3 . These are due to the much too aggressive leading edge angle. Cases 2 and 3 illustrate the importance of leading edge angle on an efficient wing design. Moderate leading edge angle, such as that in case 2, results in a wing with little separation, which should have comparable performance to the design criteria. A too aggressive leading edge angle (case 3) can result in flow separation that manifests on the pressure side of the wing, which will undoubtedly reduce performance.

Finally, we examine the results for case 4 which corresponds to a wing with dynamically adjusted leading edge angle. The leading edge angle in this case is set such that the wing leading edge always aligns approximately with the oncoming flow. This leading edge criteria is chosen for several reasons. First, we chose to align the leading edge of the wing to reduce the likelihood of leading edge flow separation. Second, we wanted to minimize the effect of camber on the pitching moment of the wing, hence, choosing just the right amount of camber can mitigate overly large pitching moments which will result in controllability challenges in practical applications. The FastAero pressure differential distribution results again illustrate the likelihood of separation is low, due to the relatively well distributed suction pressure across the suction side of the wing. The lack of strong pressure peaks on the suction side of the wing, indicates that the camber has a separation reducing effect for this infinitely thin wing. In addition, as in previous cases, the pressure differential is stronger in the outboard portions of the wing than the inboard portions. This higher pressure distribution in the outboard region of the wing is due to the reduced wing area available to produce the desired lift, and will likely encourage the generation of leading edge vortex structures. The Navier-Stokes simulations (figure 6) confirm our predictions that there is little flow separation in the dynamically adjusted leading edge case. The flow is well behaved throughout the flapping cycle with only a small leading edge 
vortex being generated at the last quarter of the downstroke and shed into the tip vortex at the end of the downstroke. This wing displays the desired aerodynamics characteristics and presents a good candidate for further study and optimization at a higher fidelity level.

\section{VI.B. Detailed Examination of the Viscous Simulations}

The viscous simulations of the efficient wings reveal some subtle, yet interesting details about the efficient flapping wing that we have designed (figure 6). First, as might be expected from the target wake circulation distribution, there is a strong tip vortex being shed during the downstroke in all of the simulations. This appears to be the primary structure during the downstroke portion of the flapping cycle. In addition, the cases that exhibit any leading edge separation, all initially form a leading edge vortex, regardless of whether the eventual flow separates fully from the wing or not. In the most extreme viscous example, case 1, we observe a strong leading edge vortex structure forming across the entire leading edge, with some spanwise convection of the vortex into the tip vortices. Similarly, in the adjusted leading edge case, we observe the formation of a leading edge vortex in the outer third of the wing, with that structure shedding into the tip vortex structure.

\section{VI.C. Force Traces: Viscous vs. Inviscid}

The time evolution of forces in each of the geometries considered sheds light onto the effectiveness of that design. The wings are each designed for a target average lift coefficient, $C_{L}=0.44$ and for a net zero horizontal force. These wings were designed for an operating Reynolds number of approximately $\operatorname{Re}=$ 30,000. FastAero does not consider viscous forces, so should predict a net positive thrust, while the NavierStokes simulations are performed at a Reynolds number of $\operatorname{Re}=3,000$, therefore, we should expect to predict net drag in this case. This discrepancy in the Reynolds number is likely to have a large impact on the horizontal forces.

We observe (Figure 8 and 10 ) that the FastAero vertical and horizontal force predictions remain relatively invariant to the camber and geometry changes. This is primarily due to the lack of viscous modeling. Therefore, the viscous effects that are present are readily observed by comparing the FastAero force trace to the Navier-Stokes simulation force trace. The individual cases are examined in the paragraphs that follow.

Case 1 indicates a significant difference between the FastAero force prediction and the Navier-Stokes simulation. The Navier-Stokes method predicts a higher vertical force during the downstroke and a lower vertical force during the upstroke portion of the wingbeat cycle. This is an intriguing result, that we attribute to the formation of a vertical force augmenting, leading edge vortex structure during the downstroke. This LEV structure is generated at the start of the downstroke, and persists above the wing through the first third of the downstroke. This is clearly observed in the simulation results (Figure 6). This LEV initially produces smooth vertical force generation as it is created and resides above the wing, but as soon as the LEV separates and the suction side of the wing is characterized by more chaotic separated flow, the force trace becomes less smooth due to the unsteady shedding. During the upstroke, we postulate that the vertical force augmentation is no longer present due to the LEV being fully shed at the end of the downstroke, and subsequently, the wing has little capacity to generate vertical forces. The horizontal force predictions (Figure 10) illustrate that the wing design in case 1 is dominated by viscous drag forces. While the inviscid and viscous horizontal force traces trend similarly, the offset between the two is large, and indicative of an inefficient design. The viscous case predicts a wing that is incapable of flight without additional thrust forces. This is due to two factors, first the Reynolds number of the viscous simulations is much lower than the design case derived in the energetics model, and second, the viscous flow separation adversely penalizes the horizontal force performance.

The time evolution of vertical forces in cases 2 and 4 illustrate similar behavior and both trend well with the inviscid predictions (Figure 8 and 10 ). This is not surprising, since the flow remains mostly attached through the duration of the wingbeat cycle for both of these cases (Fig. 6). The reason that the $10^{\circ}$ leading edge case (case 2) behaves similarly to the dynamically adjusted leading edge case (case 4) is that the angle the leading edge forms with the flow at the more extreme points in the flapping cycle is approximately ten degrees. The horizontal forces in cases 2 and 4 both show the most potential for success in a MAV application. the force histories are smooth and both show positive thrust during the downstroke. The dynamically adjusted leading edge angle case (case 4) shows the best agreement between the inviscid design 
and viscous simulations. This suggests that leading edge angle is an important consideration in flapping wing design, especially for membrane wings.

The time evolution of vertical and horizontal forces for case 3 (Figure 8 and 10) illustrate poor agreement between the inviscid and viscous solutions. Here, we imposed an extreme airfoil camber throughout the flapping cycle, so that the leading edge angle was and extreme $20^{\circ}$. As such, it is likely that the angle formed between the leading edge geometry and the oncoming flow is drastically different - with a predisposition for flow separation on the wing underside. In this case, we suspect that the stagnation streamline will actually attach to the airfoil at a location on the suction side of the wing. This means that any flow below the dividing stagnation streamline will need to turn around the sharp leading edge and will therefore cause an underwing separation bubble or complete separation to form. This is likely responsible for the observed reduction in lift and the increase in drag. In addition, the effect of too great a leading edge angle may manifest in a leading edge vortex being formed on the underside of the wing, adversely affecting the aerodynamics. In this case, the downward vertical force and drag force would both increase.

\section{Conclusions}

We have shown in this paper that our multi-fidelity computational framework is capable of developing initial candidate designs for flapping wing vehicles. In particular, we have demonstrated our ability to generate and examine wings with dynamic camber for leading edge flow alignment which results in separation suppression. We use wing twist modulation for generating the required circulation distribution. We also show, similar to previous inviscid-viscous studies, that significant insight can be gained by assessing the pressure distribution predicted by an inviscid method. Examining the pressure distributions with a critical eye for pressure spikes and subsequent strong adverse pressure gradients permits initial insight into the performance of the wing and can save significant amounts of computational effort at higher fidelity levels. Despite the significant insight that can be gleaned from inviscid methods, we are ultimately dependent on higher fidelity simulations for confirming our designs. Ultimately, our results demonstrate that inviscid design tools, used judiciously at the early stages of design, can be very effective in reducing the cost and time to design effective flapping wing MAVs.

In addition, our results demonstrate the importance of leading edge angle in efficient fliers. By prescribing a leading edge angle we are able to reduce the likelihood of flow separation, especially when the leading edge aligns with the flow. We show that leading edge angle adjustment can be performed approximately and separation can still be averted. We hypothesize that the range of leading edge angles is dependent on how extreme the flapping motions and force generation are. We expect, because we prescribe an optimal energetics condition in our design process, that wings will have reasonable performance requirements for the particular flight condition.

\section{Acknowledgements}

The authors would like to thank Hesam Salehipour for his previous contributions in energetics modeling. The results from prior research has been instrumental in this process. The authors also gratefully acknowledge the support of the AFOSR MURI program on bio-inspired flight, the AFOSR YIP program, the University of Massachusetts Lowell faculty start-up, and the computing resources of NERSC supported by the Director, Office of Science, Computational and Technology Research, U.S. Department of Energy under Contract No. DE-AC02-05CH11231.

\section{References}

\footnotetext{
${ }^{1}$ Willis, D. J., Israeli, E. R., Persson, P.-O., Drela, M., Peraire, J., Swartz, S. M., and Breuer, K. S., "A Computational Framework for Fluid Structure Interaction in Biologically Inspired Flapping Flight," 18th AIAA Computational Fluid Dynamics Conference, Miami, FL, June 2007, AIAA-2007-3803.

${ }^{2}$ Persson, P.-O., Willis, D. J., and Peraire, J., "The Numerical Simulation of Flapping Wings at Low Reynolds Numbers," 48th AIAA Aerospace Sciences Meeting and Exhibit, Orlando, Florida, 2010, AIAA-2010-724.

${ }^{3}$ Salehipour, H. and Willis, D. J., "A wingbeat kinematics influenced, energetics model for flapping flight," 48th AIAA Aerospace Sciences Meeting and Exhibit, Orlando, Florida, January 2010.

${ }^{4}$ Salehipour, H. and Willis, D. J., "A Novel Energetics Model for Examining Flapping Flight," ECCOMAS CFD 2010 conference in Lisbon, Portugal, June 2010.
} 
${ }^{5}$ Hall, K., Pigott, S., and Hall, S., "Power requirements for large-amplitude flapping flight," JOURNAL OF AIRCRAFT, Vol. 35, No. 3, MAY-JUN 1998, pp. 352-361, AIAA 35th Aerospace Science Meeting / Nonlinear Dynamical Systems Symposium, RENO, NEVADA, JAN 06-10, 1997.

${ }^{6}$ Willis, D. J. and Salehipour, H., "Preliminary Design of Three-Dimensional Flapping Wings from a Wake-Only Energetics Model," AIAA Atmospheric Flight Mechanics Conference, Toronto, Ontario, August 2010, AIAA-2010-7630.

${ }^{7}$ Willis, D. J. and Salehipour, H., "A Multi-fidelity Framework for Designing Compliant Flapping Wings," ECCOMAS CFD 2010 conference in Lisbon, Portugal, June 2010.

${ }^{8}$ Willis, D. J., Peraire, J., and White, J. K., "A combined pFFT-multipole tree code, unsteady panel method with vortex particle wakes," Internat. J. Numer. Methods Fluids, Vol. 53, No. 8, 2007, pp. 1399-1422.

${ }^{9}$ Saad, Y. and Schultz, M. H., "GMRES: a generalized minimal residual algorithm for solving nonsymmetric linear systems," SIAM J. Sci. Statist. Comput., Vol. 7, No. 3, 1986, pp. 856-869.

${ }^{10}$ Phillips, J. and White, J., "A Precorrected-FFT Method for Electrostatic Analysis of Complicated 3-D Structures," IEEE Transactions On ComputerAided Design of Integrated Circuits and Systems, Vol. 16, 1997.

${ }^{11}$ Greengard, L., The Rapid Evaluation of Potential Fields in Particle Systems., MIT Press, Cambridge, 1988.

${ }^{12}$ Appel, A. W., "An Efficient Program for Many-body Simulation." SIAM J. Sci. Stat. Comput., Vol. 6, 1985.

${ }^{13}$ Peraire, J. and Persson, P.-O., "The compact discontinuous Galerkin (CDG) method for elliptic problems," SIAM J. Sci. Comput., Vol. 30, No. 4, 2008, pp. 1806-1824.

${ }^{14}$ Alexander, R., "Diagonally implicit Runge-Kutta methods for stiff o.d.e.'s," SIAM J. Numer. Anal., Vol. 14, No. 6, 1977, pp. 1006-1021.

${ }^{15}$ Persson, P.-O. and Strang, G., "A simple mesh generator in Matlab," SIAM Rev., Vol. 46, No. 2, 2004, pp. 329-345.

${ }^{16}$ Morgan, K. and Peraire, J., "Unstructured grid finite element methods for fluid mechanics," Inst. of Physics Reviews, Vol. 61, No. 6, 1998, pp. 569-638.

${ }^{17}$ Persson, P.-O., Bonet, J., and Peraire, J., "Discontinuous Galerkin Solution of the Navier-Stokes Equations on Deformable Domains," Comput. Methods Appl. Mech. Engrg., Vol. 198, No. 17-20, 2009, pp. 1585-1595.

${ }^{18}$ Persson, P.-O. and Peraire, J., "Curved Mesh Generation and Mesh Refinement using Lagrangian Solid Mechanics," 47th AIAA Aerospace Sciences Meeting and Exhibit, Orlando, Florida, 2009, AIAA-2009-949. 


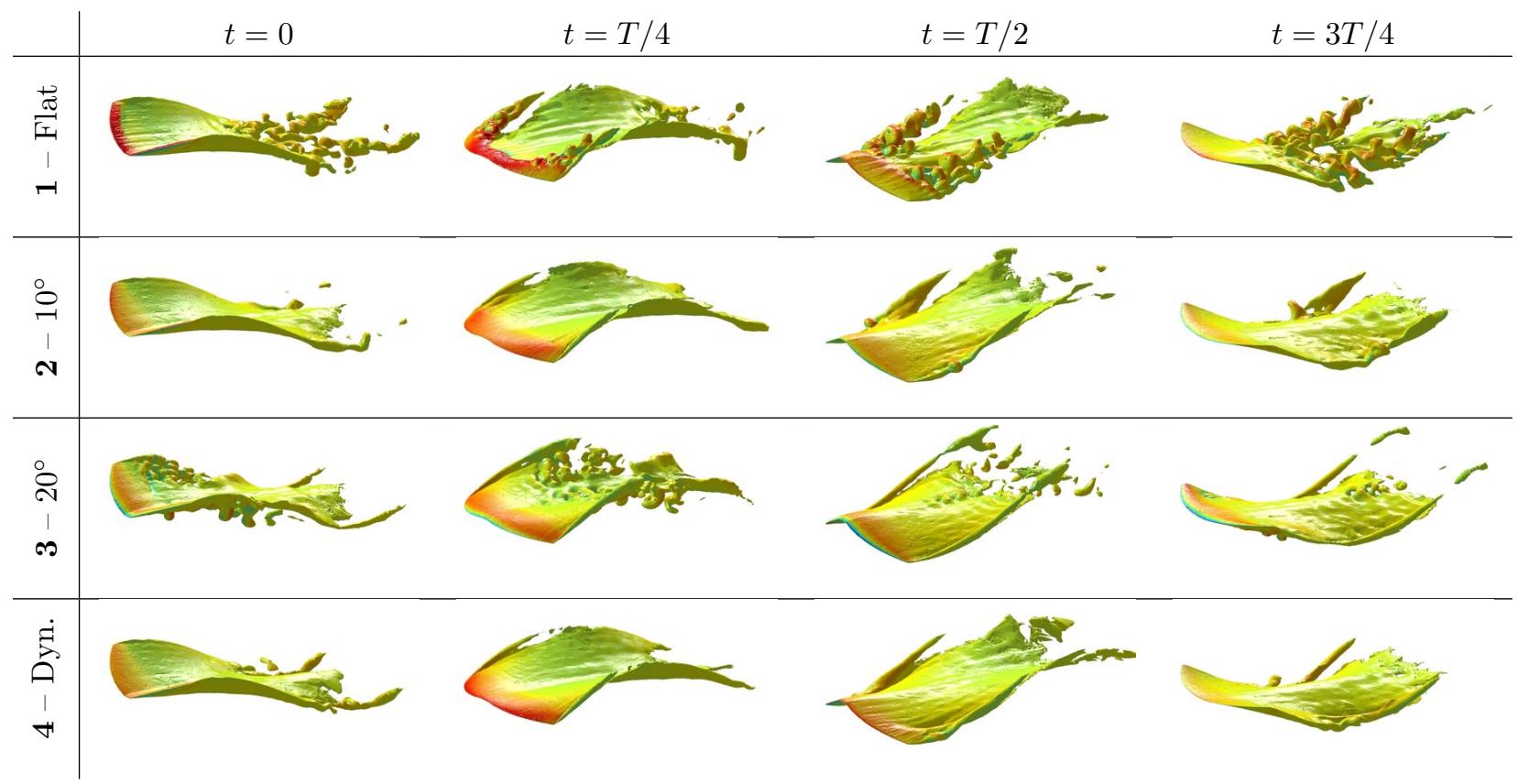

Figure 6. The flow field around the flapping wing pair computed by the $3 D G$ Navier-Stokes code, visualized as Mach number color plots on isosurfaces of the entropy. The plots correspond to the four design cases considered (top to bottom) and the times $t=0, t=T / 4, t=T / 2$, and $t=3 T / 4$ (left to right), where $T$ is the period.

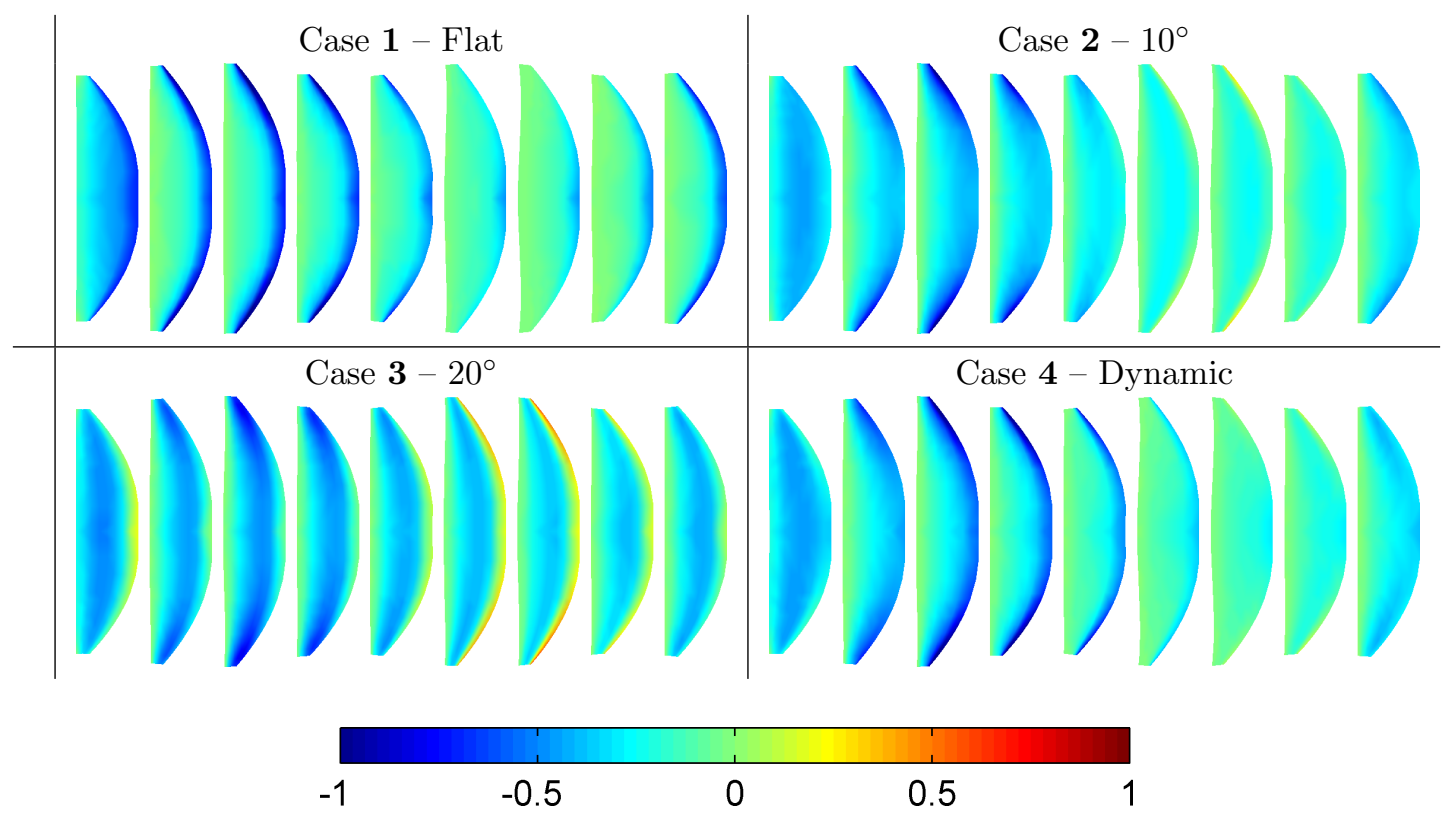

Figure 7. The surface pressure differential distribution for a complete flapping cycle predicted using FastAero for the four case studies in this paper. The wing is flyng from left to right starting with a downstroke. 

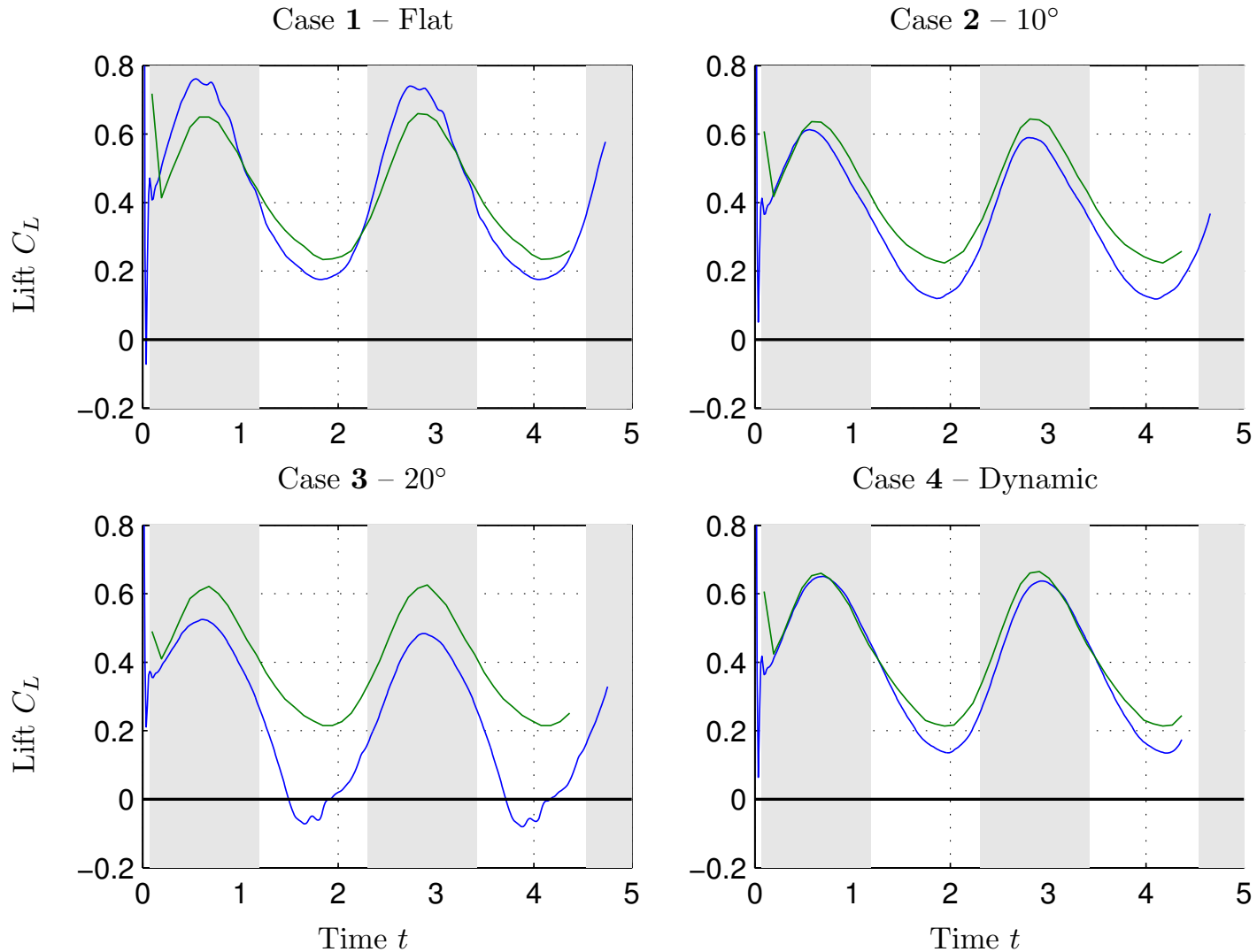

Lift, Navier-Stokes (3DG) Lift, Panel (FastAero)

Figure 8. The lift coefficients $C_{L}$ computed by the two simulation codes for the four cases.

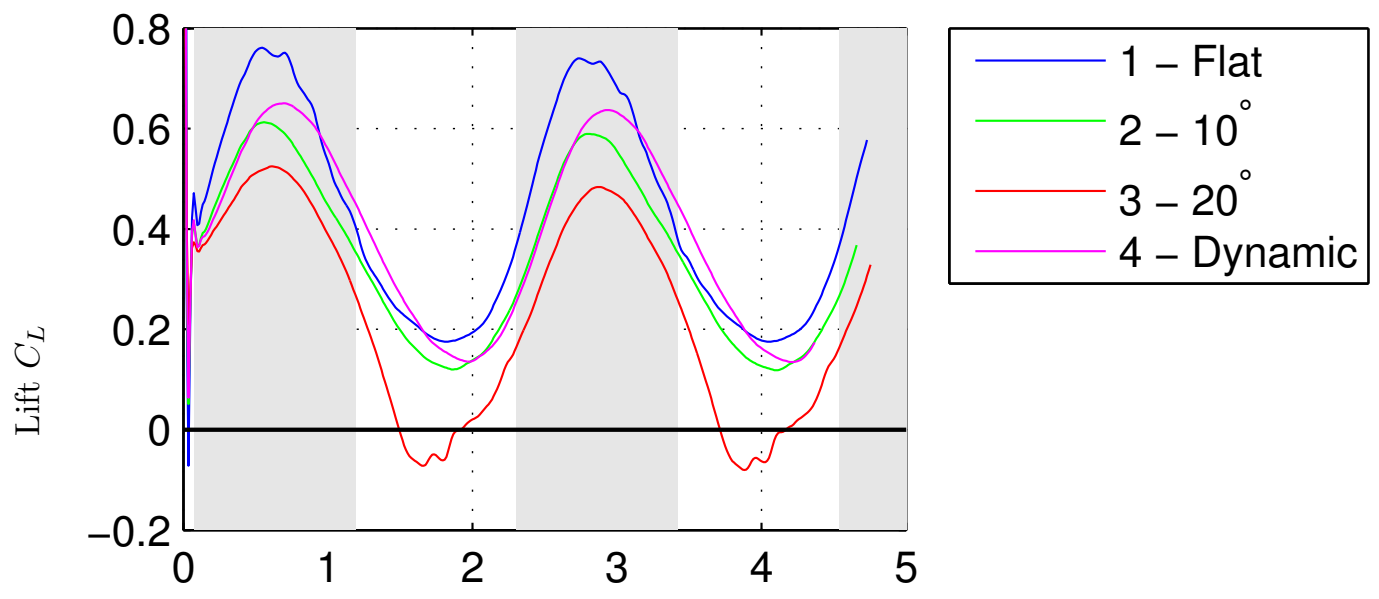

Figure 9. The lift coefficients $C_{L}$ computed by the $3 D G$ Navier-Stokes code for the four cases. 
Case 1 - Flat
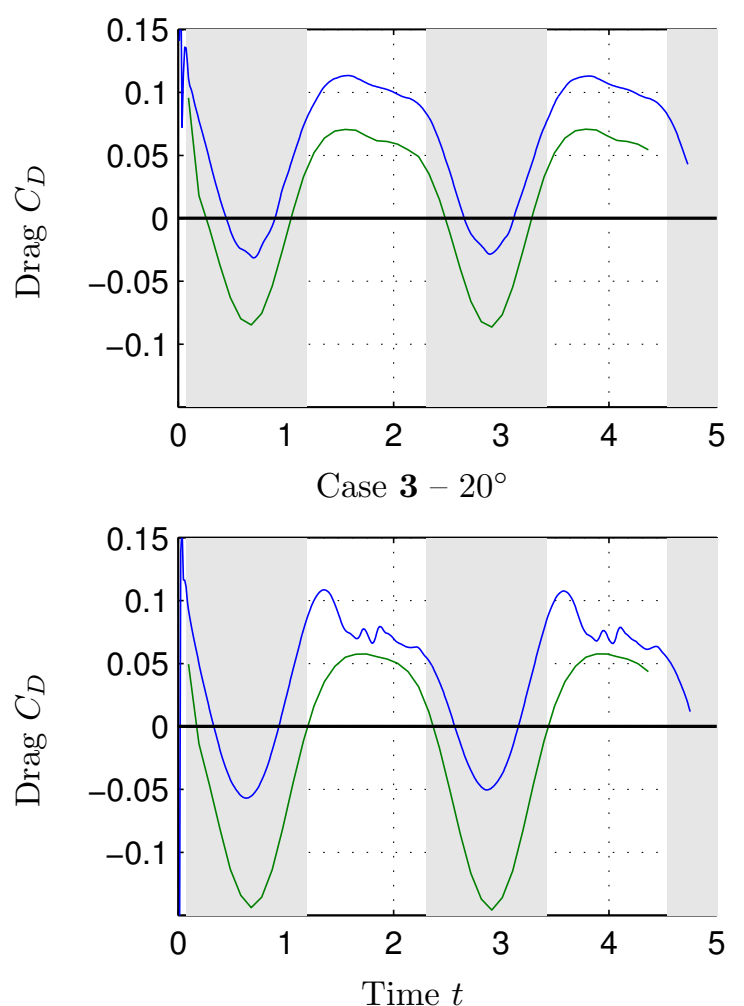

Case $\mathbf{2}-10^{\circ}$
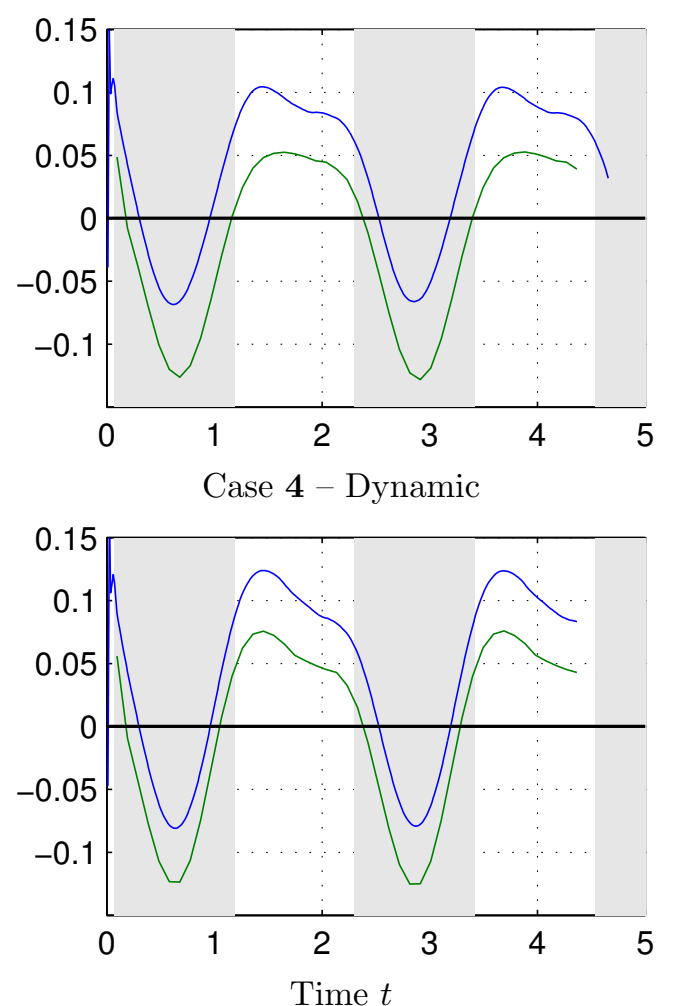

Drag, Navier-Stokes (3DG)

Drag, Panel (FastAero)

Figure 10. The drag coefficients $C_{D}$ computed by the two simulation codes for the four cases.
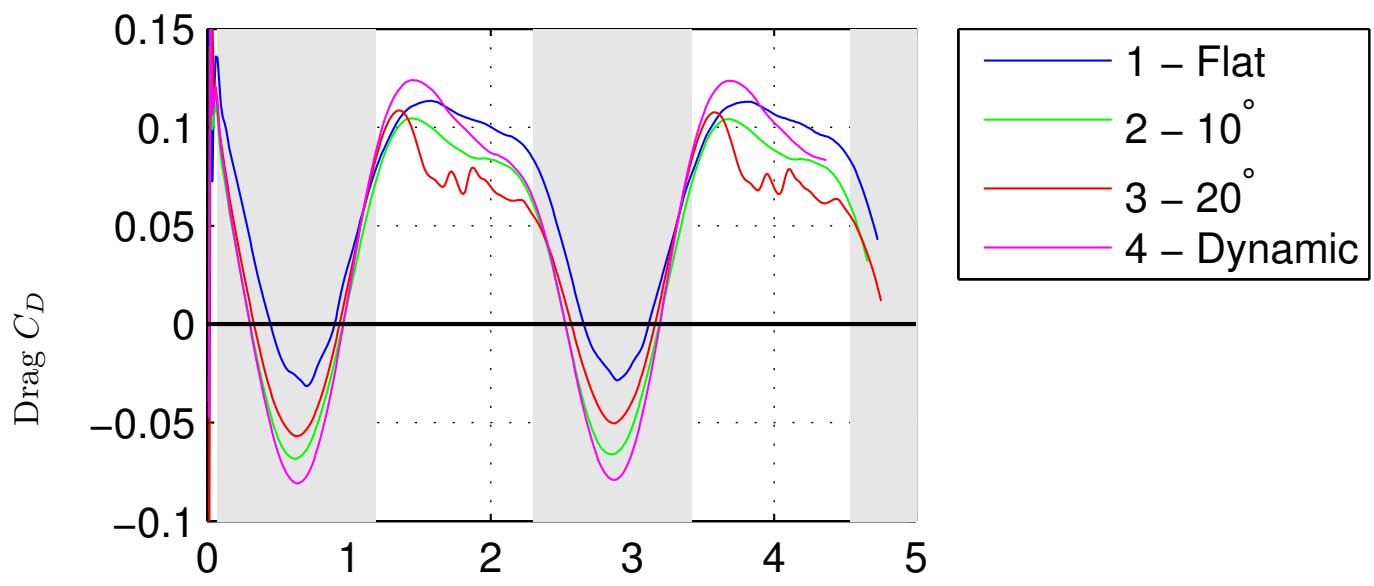

Figure 11. The drag coefficients $C_{D}$ computed by the $3 D G$ Navier-Stokes code for the four cases. 